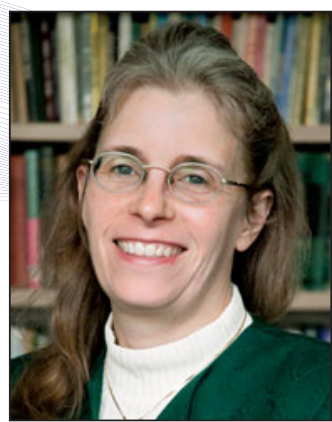

Susan Trolier-McKinstry 2017 MRS President

\title{
The impacts of publication
}

The vast majority of scientists and engineers aspire to have their contributions make a difference- to leave science and technology further ahead than where we found them in terms of fundamental understanding, improved education, and/or commercial viability. Publications, in journals and patents, provide opportunities to make an impact beyond the course of our individual careers. So how should we (and our department heads, managers, program managers, etc.) assess the value of these contributions?

There are multiple ways of measuring the impact of a publication, from the change in the way the scientific community understands some phenomenon, to impact factor, to the citation half-life, to the total number of citations garnered over the lifetime of a publication, to the economic value resulting from the work. Each of these metrics measures different factors, so it is not surprising that while a body (or piece) of work may fare well on one scale, it might fare less well on another.

Consider the seminal work of Sessler and West on electret microphones, for example. While their key paper, "Self-Biased Condenser Microphone with High Capacitance" (J. Acoust. Soc. $A m .34$ (11), 1787 [1962]), lays out the fundamentals of this technology, it has barely more than 100 citations, and yet the resulting technology is ubiquitous. Another excellent example is the classic paper by Oliver and Pharr, "An Improved Technique for Determining Hardness and Elastic Modulus Using Load and Displacement Sensing Indentation Experiments" (J. Mater. Res. 7 (6), 1564 [1992]). This paper would have had an impact factor of only 12.5 in its first two years, yet now has nearly 12,000 citations (based on the citation report in the Web of Science Core collection) and is cited more than 450 times per year. This is a paper where the citation half-life is measured in multiple decades, as the techniques developed have become staples in the materials science repertoire. As a final example, in modern academia, the impact factor of a candidate's publications is widely used in hiring, promotion, and tenure decisions, whereas the economic value or long-term scientific impact of a body of work may not be assessed as directly.

Thankfully, we as a community are accustomed to utilizing data from multiple sources to assess what we can learn from various theoretical and experimental techniques. Failure to look critically at all of the data would be an injustice. I would argue that the same is true in looking at the impact of any scientist or publication on the field. We, as scientists, need to assess the quality of the science or technology that we/our colleagues produce. It may take more time, but it is what we are trained to do.

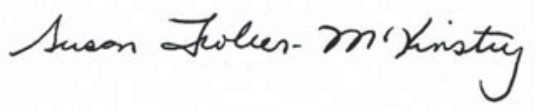

